\title{
Evaluating Models of the Muonium-to-Antimuonium Transition
}

\author{
By investigating models of physics beyond the standard model, \\ researchers determine the parameter spaces where future experiments \\ might detect-or rule out-a new interaction.
}

By Erika K. Carlson

$\prod$ he exotic atom muonium consists of an antimuon and an electron bound together in a hydrogen-like state. If muonium were to transition into antimuonium, consisting of a muon and a positron, the reaction would violate lepton flavor number, which is conserved in the standard model of particle physics. Thus, detecting a

muonium-to-antimuonium transition would be a clear sign of physics beyond the standard model. Now, in light of recent experimental results, Takeshi Fukuyama of Osaka University and colleagues have examined several models of beyond-standard-model physics and investigated the predictions they make about the muonium-to-antimuonium transition [1]. Their study should inform the analysis of planned experiments to search for signs of this transition in the near future at facilities in Japan and China.

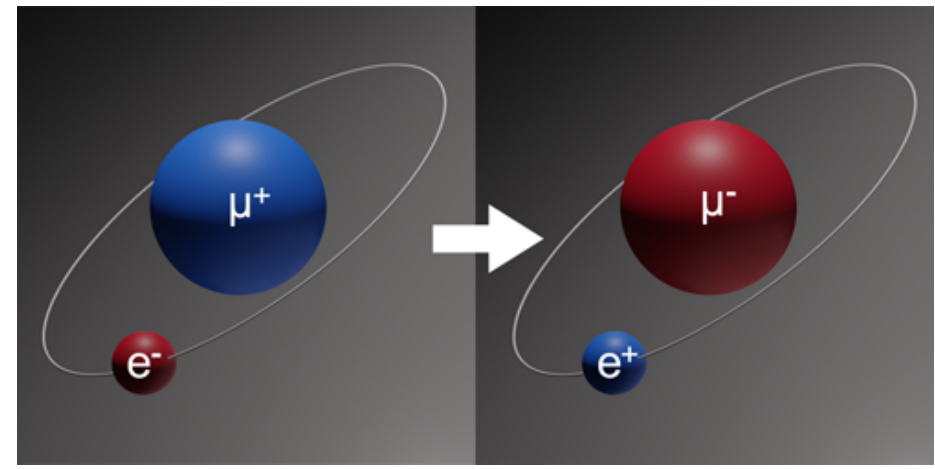

Credit: APS
The last experiments directly investigating the muonium-to-antimuonium transition occurred in the late 1990s. However, experiments in the twenty-or-so years since have produced results that relate to this transition indirectly, such as measurements of neutrino-oscillation parameters and constraints on new particles predicted by the same lepton-flavor-number-violating models that predict the muonium-to-antimuonium transition. Based on these recent experimental results, Fukuyama and colleagues evaluated several models of physics beyond the standard model and determined the parameter spaces where future experiments might detect-or rule out-the transition for a given model. For example, of a class of models known as radiative neutrino mass models, the team found that one called the Zee-Babu model could produce the largest probability of a muonium-to-antimuonium transition occurring. Planned experiments to search for the transition at the Japan Proton Accelerator Research Complex and the China Spallation Neutron Source could test such models.

Erika K. Carlson is a Corresponding Editor for Physics based in New York City.

\section{REFERENCES}

1. T. Fukuyama et al., "Models of the muonium to antimuonium transition," Phys. Rev. D 105, 015026 (2022). 